Patienten, die nach strikten ESUS-Kriterien eingeschlossen wurden. Sie erfüllten die MRT/CT-Kriterien für einen embolischen Infarkt und hatten eine unauffällige Echokardiografie. Bereits nach einem Jahr konnten die Ärzte bei $24 \%$ der Patienten ein VHF nachweisen. Geht man von einem ähnlichen Verlauf wie bei Crystal-AF aus, entspreche dies rund $60 \%$ der Patienten nach drei Jahren, und der Anteil wäre sogar noch höher, würde man die etwas lockerere Definition für VHF aus der US-Studie übernehmen: Dort genügte eine Episode von 30 Sekunden für ein VHF, in der Studie von Schäbitz waren es mindestens zwei Minuten. In Crystal-AF lagen etwa $30 \%$ der VHF-Episoden im Zeitfenster von weniger als zwei Minuten.

Schäbitz führte die Unterschiede zur US-Studie auf die striktere Anwendung der ESUS-Kriterien mit klarem Ausschluss nicht embolischer und kardialer Ursachen zurück. Auch seien die LoopRekorder früher implantiert worden als in Crystal-AF - im Schnitt 20 Tage nach dem Insult.

\section{Haustier-VHF?}

Wenn nun die meisten ESUS-Patienten ein VHF entwickeln und die meisten $\mathrm{Pa}$ tienten mit VHF eine orale Antikoagulation erhalten, könnte man sich den Umweg über das aufwändige und für die Masse der Patienten sowieso nicht praktikable kardiale Langzeit-Monitoring eigentlich sparen. Es wäre dann wohl

\title{
Enzephalopathie durch Herbstzeitlose
}

Colchicin aus den Blättern der Herbstzeitlose zählt zu den wirkungsvollsten Zytostatika. Es wird gelegentlich gegen Gicht eingesetzt. Auf einen Selbstversuch mit Herbstzeitlosen sollte sich aber niemand einlassen. Dies demonstrierte eindrucksvoll eine 47-jährige Frau, die im Selbstversuch mit zwei Blättern der Herbstzeitlose ihre Psoriasisarthritis lindern wollte. Kurz darauf kämpften die Ärzte auf der Intensivstation um ihr Leben. In den ersten Stunden nach dem Genuss der Blätter musste sie sich erbrechen, nach und nach quittierten einzelne Organe ihren Dienst. Neurologen um Dr. Wolf-Dirk Niesen vom Universitätsklinikum Freiburg fanden unter anderem eine ausgeprägte Panzytopenie infolge einer Knochenmarksdepression, eine Enzephalopathie mit Psychosyndrom und ausgeprägten Lähmungen sowie eine axonale Polyneuropathie und Myopathie. Hinzu kam eine Kleinhirnblutung sowie ein beatmungspflichtiges ARDS. Die Patienten konnte gerettet werden. Eine Colchicinvergiftung kann sowohl direkt als auch indirekt bedingt durch ein Multiorganversagen schwere neurologische Defizite hervorrufen, folgern die Freiburger Neurologen.

Thomas Müller

Poster 121: Bornkamm K, Niesen WD. Selbsttherapieversuch mit Blättern der Herbstzeitlosen - das Vollbild einer Colchicin-Intoxikation. 33. ANIM-Tagung, Berlin, 28.-30.1.2016

sinnvoll, gleich nach einem ESUS mit der Antikoagulation zu beginnen. Der Neurologe hält jedoch dagegen, dass es vielleicht immer noch einen Anteil von $30-40 \%$ der ESUS-Patienten gibt, die auch langfristig kein VHF entwickeln. Sei es also vertretbar, diese den Risiken einer oralen Antikoagulation auszusetzen, zumal die Rezidivrate bei ESUS recht gering ist? Auch müsse man sich die Frage stellen, welche klinische Bedeutung ein viele Monate nach einem Infarkt auftretendes VHF überhaupt habe. Vielleicht handle es sich hierbei eher um ein „Haustier-VHF“. Antworten könnten in naher Zukunft Studien wie RE-SPECT ESUS und NAVIGATEESUS liefern, die bei jeweils über 6.000 Patienten eine Antikoagulation mit DOAK versus ASS-Therapie zur Schlaganfallprophylaxe prüfen. Bis dahin könne man sich vielleicht an VHF-Risikofaktoren orientieren: So hätten in der eigenen Studie vor allem ältere Patienten, solche mit einem $\mathrm{CHA}_{2} \mathrm{DS}_{2}$-VASc-Score über 5, einer Leukoaraiose oder einem Infarkt im Mediaversorgungsgebiet ein VHF entwickelt.

Thomas Müller

Symposium Vorhofflimmern und Schlaganfall, 33. ANIM-Tagung, Berlin, 28. - 30.1.2016

\section{Aneurysmen clippen oder coilen? - Das hängt von Alter und Anatomie ab}

\section{Das Clipping von rupturierten subarachnoidalen Aneurysmen hat zwar viele Vorteile, ist aber nicht für alle die beste Wahl. Vor allem junge Patienten und solche mit breitbasigen Aneurysmen scheinen häufiger von einem chirurgi- schen Eingriff zu profitieren.}

nzwischen haben drei randomisiertkontrollierte Studien leichte Vorteile bei der Morbidität und Mortalität für das endovaskuläre Coiling von geplatzten subarachnoidalen Aneurysmen ergeben. So waren in der größten der drei Studien, der International Subarachnoid
Aneurysm Trial (ISAT) mit über 2.100 Patienten, nach einem Jahr 23,5\% verstorben oder zeigten deutliche Behinderungen, bei solchen mit neurochirurgischem Clipping knapp $31 \%$. Nach zehn Jahren waren $15 \%$ der Coiling-Patienten und $17 \%$ derjenigen mit Clipping deut- lich behindert (mRS 3 - 5) und 17 versus $21 \%$ waren verstorben (mRS 6).

Auch in den anderen beiden, deutlich kleineren Studien zeigte sich ein Vorteil für die endovaskuläre Therapie, erläuterte Privatdozent Nima Etminan von der neurochirurgischen Universitätsklinik in Mannheim. Allerdings kam es mit dem Coiling deutlich häufiger zu erneuten Blutungen, vor allem wenn die Aneurysmen $\mathrm{zu}$ weniger als $75 \%$ verschlossen werden konnten. Unterschiede ergaben sich in Subgruppenanalysen der Studien auch beim Alter der Patienten, der Lokalisation und der Anatomie der Aneurysmen, sodass letztlich individuell entschieden werden müsse, welches 